\title{
Service users' experiences of residential alternatives to standard acute wards: qualitative study of similarities and differences
}

\author{
Helen Gilburt, Mike Slade, Diana Rose, Brynmor Lloyd-Evans, Sonia Johnson and David P. J. Osborn
}

\section{Background}

Little is known about the preferences and experiences of people with mental illness in relation to residential alternatives to hospital.

\section{Aims \\ To explore patients' subjective experiences of traditional hospital services and residential alternatives to hospital.}

\section{Method}

In-depth interviews were conducted with 40 purposively selected patients in residential alternative services who had previously experienced hospital in-patient stays. Transcripts were coded and analysed for thematic content.

\section{Results}

Patients reported an overall preference for residential alternatives. These were identified as treating patients with lower levels of disturbance, being safer, having more freedom and decreased coercion, and having less paternalistic staff compared with traditional in-patient services. However, patients identified no substantial difference between their relationships with staff overall and the care provided between the two types of services.

\section{Conclusions}

For patients who have acute mental illness but lower levels of disturbance, residential alternatives offer a preferable environment to traditional hospital services: they minimise coercion and maximise freedom, safety and opportunities for peer support.

\section{Declaration of interest}

None.
Although treatment and containment remain at the heart of UK psychiatric in-patient care provision, ${ }^{1}$ patient experiences during admission are of increasing importance. Studies of traditional psychiatric hospital admission have often highlighted patients' poor experiences, ${ }^{2}$ which has led to a number of calls for alternatives to hospital. ${ }^{3,4}$ The development of community-based services has partly met this need, but there is wide recognition that residential admission is still a requirement for a small proportion of patients. Residential alternatives to hospital have a long history in both the UK and USA. A recent survey identified that a substantial proportion of in-patient care was being provided by such institutions in England, ${ }^{5}$ yet little research has been undertaken into patients' experiences within these services and whether these services are a viable alternative to hospital from the perspective of the people using them. Since these services aim to provide a different model of care to traditional services, we undertook a qualitative study to understand how admissions to alternative in-patient services are experienced, and how this experience compares with traditional hospital services. The aim was to access the subjective experience and patient values influencing the patient experience which may be specific to alternative services.

\section{Method}

Participants were recruited from six residential alternatives to hospital. These comprised two crisis houses run by the voluntary sector and staffed by non-clinical staff, one crisis house run by the statutory sector and staffed by social care and clinical staff, one crisis house staffed by clinical staff alone, one brief admission unit based in a hospital, and a hospital service where all staff were trained in and using the Tidal Model. ${ }^{5}$ The services covered geographically urban and rural areas and were representative of the five different types of service identified by a national study of residential alternatives to hospital. ${ }^{6}$

Purposive sampling was used to maximise the likelihood of obtaining a complete range of views. Eligible patients were those aged 18-65 years who had had a previous admission to a standard acute hospital and who were able to provide informed consent. Eligible patients were identified by staff in each service; after asking the ward staff for consent to contact these patients, the researcher approached the eligible patients and invited them to participate in the study.

\section{Data collection}

Data collected for the whole sample included age, gender, ethnicity and legal status. In-depth interviews of up to $2 \mathrm{~h}$ duration were conducted with six patients in each service. A topic guide was used for the in-depth interviews, based on previous research investigating what service users identified as important in defining their experience of hospital admission. ${ }^{7}$ The topics were relationships, safety, treatment, freedom, ethnicity, religion and environment. The interviewer asked participants individually to comment on their stay in the alternative service in which they were currently resident and on their previous hospital admissions. All interviews were conducted at the respective residential alternative service by one researcher (H.G.).

\section{Statistical analysis}

The in-depth interviews were audiotaped and transcribed. Manuscripts were imported into QSR NVivo 7 for Windows (www.qsrinternational.com). We analysed the material using a thematic analysis. ${ }^{8,9}$ Initially four interviews were independently coded and an open coding session between four researchers was used to confer on and list themes. These themes were used as a basis to code the rest of the transcripts, with new themes being 
added as they emerged. Findings and emerging themes were tested for validity through discussion - in both one-to-one and group meetings - by an interdisciplinary team comprising researchers with psychiatric, psychological and social work backgrounds, both with and without clinical experience and experience of using mental health services. Furthermore, efforts were made to explore the interviewees' underlying reasoning and elements within the data that appeared to contradict the emerging themes ('deviant case analysis').

\section{Results}

Ten main themes emerged from the analysis of patients' accounts of their experiences of admission, which highlighted perceived differences and similarities between alternative and traditional in-patient services. In addition a further theme was drawn out where patients noted opinions and preferences for different types of services. Selected quotations are presented in the following text to illustrate each theme (additional illustrative excerpts from interview transcripts are provided in online supplement 1).

The hospital-based alternative using the Tidal Model had poor implementation of the specific model of care, and functioned as a standard acute admission ward. ${ }^{10}$ It is therefore considered in this analysis to be equivalent to hospital in-patient services in terms of patient experiences.

'But it's not employed. You're talking about the Tidal Model, aren't you? You are supposed to sit down each day . . . yeah, and they don't do it.' (Tidal Model service 1102)

\section{Opinions about services}

Twenty-five patients expressed an overall opinion about one type of service. Seventeen patients reported that their overall experience of an alternative service had been positive, whereas two had negative experiences of alternative services. Of those who expressed an opinion about hospital, eight labelled their experience overall as negative and one patient had a positive experience of hospital.

'I think I would prefer to come here because the environment is a lot more calm, a lot more easy-going.' (Alternative service, 3140)

'It was a total and utter nightmare, I've never experienced anything so extreme with people running up and down corridors, screaming at 3 in the morning, I was terrified. would never go there again. Hospital would make me worse.' (Hospital service, 6122)

\section{Relationships}

Relationships were the most frequently reported theme in the experiences of patients, and featured in all of the transcripts. In addition, the majority of the other themes were reported within the context of relationships experienced while in hospital. Relationships focused on were those formed between staff and patients; patients among themselves; and with family and friends outside the hospital. Between staff and patients, effective communication was identified as a key factor in building successful relationships. Staff characteristics identified as important were qualities such as being nice, caring, friendly, polite and genuine. These characteristics were valued by over half of the patients interviewed. Relationships had a powerful impact on patients both in alternative and traditional services but there was no clear difference in terms of the number and type of interaction between types of services. Indeed, within individual services patients highlighted staff who had particularly good communication skills and valued qualities, as well as those who lacked them.

'They [staff] were just really friendly and caring and lovely.' (Hospital service, 1164)
'They were rude, their job was to, there was, although people were sick, right, they thought everybody was stupid. They'd talk down to us like we were lower than them. (Hospital service, 2142)

Continuity of care was noted as important by three-quarters of patients. This also influenced patients' experiences of services. Many patients had used services several times. They described having established relationships with staff and with such services that positively affected their experiences of the service. It was particularly noted in some alternative services where community agencies continued to provide care despite the patient occupying an in-patient unit

'I trust the staff $100 \%$ because I've been coming here for the last, well the last $3 \frac{1}{2}$ years.' (Alternative service, 4111)

Continuity of care also extended to family and friends. A third of patients placed particular importance on staying in contact, whether by telephone or with visitors. In alternative services, patients identified that family and friends also had some impact on their care such as being able to refer directly into the service, influencing length of stay and providing information for the service. This was not noted in traditional hospital services.

'My dad has extended it [length of stay] so it will be, I will be here for the rest of today and I don't know how long then but it will do me good, I will benefit from it.' (Alternative service, 2108)

\section{Patients}

Over half of respondents identified that people treated in hospitalbased in-patient services displayed more acute illness and disturbed behaviour. This included respondents in the hospitalbased alternative services. These more acutely unwell patients contributed negatively to respondents' experience of hospital, by raising fears about safety and by making increased demands on staff time and input. Patients identified the importance of being able to relate to other patients in both a social and a therapeutic way, and that more acutely ill patients did not fulfil or negatively affected this relationship.

'There were so many people and they had lots of problems of their own and it was hard to get on and maybe talk to staff because there were so many patients in the hospital unwell.' (Hospital service, 6105)

'I would say that every other patient, most of them have been too ill to engage in relationships with.' (Hospital service, 1161)

\section{Coercion}

Reports of coercion were greater in hospital services. Instances of hard coercion, such as control and restraint, and forced medication were limited to hospital-based services, including both hospital-based alternative services. Soft coercion in the form of threats, perceived force and perceived punishment were experienced in both services but predominated in traditional hospital services. The nature of soft coercion also varied between different types of service. Patients in alternative services reported feeling coerced regarding issues of well-being such as eating and washing, and punishment took the form of a verbal reprimand. In contrast, patients in hospital more often reported punishment in terms of actions, such as loss of freedom or increased observations.

'Yeah, forced, the medicine. They say if I don't take the tablet they were going to inject me.' (Hospital service, 6106)

'Well, they force me sometimes as they do with the rest of the patients, to keep cleaner, to have a shower, have a shave and do the laundry, that kind of thing.' (Alternative service, 1143)

\section{Freedom}

Patients reported greater amounts of freedom in alternative services. Both detained and voluntary patients reported less 
freedom in traditional hospital services. Freedom incorporated being able to 'come and go' but also being able to go out and smoke a cigarette. Not being able to go out was experienced predominantly by patients in traditional hospital services. Exceptions in alternative services included restrictions for patients detained under the Mental Health Act and at the first few days of admission at alternative services.

'You have more freedom here because you can go in and out as you please, do you know what I mean, it's not like in hospital . . . I don't like to be in hospital, I prefer being here, it's much better because you've got more freedom.' (Alternative service, 2123)

'You feel like a prisoner, that isn't safe, you have to escape. That's why I did my running (AWOL) because even [if] you are not on a section, but say having a bad day and you got to the nurses and say can I just go have a walk round the grounds the answer would be no.' (Hospital service, 4102)

\section{Paternalism}

Paternalism by staff was identified by almost half of patients in both alternative and traditional hospital services. Negatively perceived paternalism was reported most frequently in traditional hospital services. Paternalism was experienced negatively when patients were treated as if they were children in terms of punitive measures, such as having their telephone taken away, or in terms of practical measures involved in running a service, such as being told when to go to bed. Patients described feeling anger and expressing aggression towards such forms of paternalism. The form of paternalism that patients experienced positively was that in which they felt cared for, e.g. fed, supervised through the night.

'I just felt as though I was back at school still, you know what I mean, having to go there and having to do something with, do pottery or something like that, I didn't want to do it. I felt like an imbecile.' (Hospital service, 3108)

'They literally treat you like babies in here. They feed you a two-hourly basis which is nice ... they watch what you eat and they'll make sure that you are looking after yourself. That's how it should be.' (Hospital service, 1125)

\section{Safety}

Safety was a dominant theme, with over half of interviewees reporting fearing for their safety during an admission to a hospital-based service and four people during an admission to a crisis house service. The most commonly reported negative influence on safety was being around people who were acutely unwell.

'For the first few days I was crying to go home because I was scared... of what I was seeing, like some people were really ill and that was quite scary.' (Hospital service, 3140)

Incidents of sexual, physical and verbal assault were directly linked to perceptions of safety in in-patient services. A number of patients experienced such incidents but witnessing them also had profound negative effects on patients' perceived safety. With one exception, all instances of assault were limited to traditional hospital services. Most commonly physical assault was reported by over a quarter of patients experiencing or witnessing attacks by patients on other patients, or instances of restraint undertaken by staff.

Patient: 'Nurse X broke someone's arm . . . under restraint . . . before I got here.' Interviewer: 'How does that make you feel?'

Patient: 'Scared of Nurse X.' (Hospital service, 1112)

Over a quarter of patients reported feeling threatened or being threatened, and these accounts were specific to hospital-based alternative and traditional services. The most commonly experienced threat was from other patients. Poor communication characterised threatening relationships with staff. Staff who were experienced as not listening or caring were perceived as oppressive and threatening. A small minority of patients reported feeling directly intimidated by staff and threatened with violence.
'They want your cigarettes all the time, I'm not the only one, they do it to other people as well. They say there are people and they are going to beat you up or something.' (Hospital service, 5138)

'I felt the whole environment was very, very threatening ... the nurses refusing to listen or understand.' (Hospital service, 1161)

Over half of patients reported feeling safe while an in-patient, the majority of these being in alternative services. Both staff and environment were key factors in patients' perceptions of safety. Staff being around and providing a sense of control through maintaining rules and boundaries was highlighted as important in giving a sense of safety, in addition to developing relationships with patients through talking to and providing help for them. Homely environments that enabled patients to have a sense of privacy while still having people around were perceived as particularly safe.

Interviewer: 'What makes you feel safe?'

Patient: 'Well, the staff are around, the surroundings are very homely, very relaxing as you would find in your own home.' (Alternative service, 2108)

A strong emphasis on feeling safe was expressed by interviewees who identified themselves as high risk in terms of suicide or harm to others. Interviewees placed particular value in containing environments such as locked doors and having people around who were able to handle high levels of distress.

'I've had times when I probably haven't been safe with myself and with my thoughts and things, and it's, I mean there's always staff around and people around if you are feeling like that whereas like at home and stuff there wasn't . . . and my family find it difficult to cope with really.' (Alternative service, 3101)

\section{Activities}

In terms of day-to-day activities, patients reported no substantial difference between alternative and traditional in-patient services. Both were characterised by a relative lack of activities with media-oriented activities such as watching television most prominent in both types of services. Access to occupational therapy was sparse in traditional services and in alternative services the activities reported were similar to those in traditional hospitals, being initiated either by service users themselves or by care staff.

'I do get bored, it's one of the reasons I go to bed in the afternoons.' (Alternative service, 4101)

\section{Treatment}

Two-thirds of patients identified medication as the primary form of treatment in both alternative and traditional in-patient services. Hospital-based services provided fixed periods when medication was dispensed, but alternative services were typically more flexible, with self-medication and provision by community-based agencies. A range of preferences was expressed by patients for different approaches. All traditional services and all but one alternative service had access to medical staff; however, where access was limited some patients expressed concerns and such input was identified as important.

Patient: 'I've felt better than I have done for a while, coming down here . . . I feel like I've sort of achieved more coming to this place.'

Interviewer: 'What has helped you achieve something while you've been here?' Patient: 'It's this new, the clozapine in particular.' (Alternative service, 4101)

'At least in Hospital $X$ they can give you something to calm you down or anything like that because it's written up if you need it, but here there's nothing like that ... It's a shame they can't help you when you need something to calm you down because there are no doctors here.' (Alternative service, 3140)

Social problems were identified by patients as key stressors both on admission to hospital and also during their stay. Over half of all patients highlighted help with social factors to be important in receiving in-patient care. Interviewees in alternative and traditional hospital services reported both positive experiences of social care provision, and also areas that were lacking. 
'I trust them [staff] enough to tell them about my immigration problems so now they are taking care of it.' (Alternative service, 2146)

Physical health problems were also raised by a quarter of patients. The most commonly reported problem was that of substance misuse. Positive action for physical health problems was described in both alternative and traditional service settings. However, where problems were reported with services these were specific to hospital-based services, and related to the interviewee's role as a mental health patient and also to a lack of skills and experience by psychiatrically trained staff.

'And they are not having their lithium checked regularly because they can't take blood.' (Hospital service, 1164)

\section{Environment}

Individuals' preferences for physical environments varied, with no clear divide between alternative services and hospital settings. There was a general appreciation of places that were 'a bit more like home', but almost half of patients referred rather to 'people make a place'. The physical environment of either type of service was not identified as being as important as the staff, other patients, their relationships and impact on the environment in providing somewhere both peaceful in terms of atmosphere and noise yet still having people around to socialise with. Characteristics of physical environments that were valued included having one's own decent-sized room, having access to outside space, some access to privacy and the overall cleanliness of the setting.

Interviewer: 'What about the environment here?'

Patient: 'Yeah, it's pretty good. It's quiet most of the time and staff come and sit with you in the sitting room sometimes and that and you can have, you know, just chat and that.' (Alternative service, 6105)

\section{Findings for ethnic minority patients}

Sixteen patients were interviewed from minority ethnic groups. Eight identified themselves as Black African or Black Caribbean, four as Black other, three as mixed race and one as Mediterranean. Ethnic minority patients did not mention qualitatively different views to other patients. Particular issues arose around safety and discrimination, with some young African-Caribbean men reporting that they could be perceived as aggressive and threatening when ill; a number of men and women reported expectations of discrimination in in-patient services. Services specifically for the African and Caribbean community were valued as places where these expectations could be put aside. It was unclear whether this led to a preference for these services, because although they catered for the Black community as a whole, respondents noted that their range of heritages - including mixed heritage - were not fully covered by these services.

Patient: 'So this organisation where, obviously that's the whole purpose of it to have people of your culture or nature or whatever to understand so you've got no reason to complain, oh they are not helping me because of your culture or your colour or whatever.

Interviewer: 'Is there anything you prefer about hospital?'

Patient: 'You get a mixture of people, you don't just get one type. In here you just get Black people, I've noticed.'

Interviewer: 'And you'd prefer a mixture? Why's that?'

Patient: 'Because I get along with them, my daughter is mixed race.' (Alternative service, 2144)

\section{Discussion}

The development of alternatives to hospital has been seen as a potential approach to the improvement of mental health services both in the USA and a number of European countries. ${ }^{11}$ Although research and service development have expanded the role of community care, there has been lesser development of residential alternatives. Offering non-clinical settings, non-clinical staff, shorter admissions and specific models of care, these alternatives have sought an improvement in patient experiences and outcomes. Studies of residential alternatives have largely concentrated on the populations served and outcomes. ${ }^{12,13} \mathrm{~A}$ study of a women's crisis house in north London found that this service was valued by patients for its environment, the absence of disturbed male patients, the availability of staff to work through past and present problems and a supportive atmosphere. ${ }^{14}$ However, this remains an isolated study of an individual service, and services established as alternatives to hospital have yet to demonstrate whether they reflect the needs and values of patients who would otherwise be admitted to hospital. This study is the first of its kind that aims to understand not only the acceptability of alternative services to service users but also how patients' values and needs are reflected in the service structure and care provided. Using a set of defined experiences established to be of importance and value to service users admitted for in-patient treatment, the research undertaken here identifies that although the majority of patients prefer such environments, the difference in preferences is more complex and lies not only in what is done and where, but also in how it is done, which has implications for both the provision of alternatives to hospital but also more widely for traditional hospital in-patient wards.

Many of the differences between hospital-based services and non-hospital alternatives in terms of what is done might be accounted for by differences between the people admitted to each type of service. The advent of developments in the provision of community care in the UK has seen hospital in-patient populations having increasingly acute illness, ${ }^{15}$ and the strength of the links made here between sharing space with disturbed patients and levels of safety highlight its very real impact on other patients. Hospital environments may further increase patients' sense of insecurity and create environments of fear and aggression through decreased levels of freedom. Key mechanisms highlighted, both in this study and in other research, ${ }^{16}$ include wards routinely locking their doors, a lack of outside space and the use of coercive measures such as control and restraint. Ironically, physical restraint when used to contain situations can create a sense of fear and distrust not only in the person restrained but also in those witnessing this action. ${ }^{17}$ By selective admission of people in crisis with chronic mental health problems and a low risk profile, services are able to offer greater levels of safety and freedom while promoting a therapeutic space for recovery. The recommendation that highly disturbed patients be treated separately from those with lesser problems has been made in the UK. ${ }^{18}$

The development of in-patient care primarily within the National Health Service (NHS) has sought both to develop specialist services and to draw on the supporting structures of other professions and specialties within the healthcare system. Yet service users identify that although non-hospital services themselves may not be able to offer such diversity of services, they have been proactive in seeking this input through liaison and partnership with other agencies, including the NHS itself. Activities and treatments on offer were seen to differ little between both types of services, a finding replicated using standardised measures of content of care. ${ }^{19}$ However, alternative services were instrumental in maintaining links for the provision of medical intervention with community treatment agencies such as assertive outreach and crisis intervention teams during admissions. This linkage led to satisfactory discharge planning and transfer of care between agencies in several instances. Such continuity of care was highly valued by service users. The relative clinical inexperience of staff in non-hospital alternatives may paradoxically have further benefited patients, given their reports of superior physical health 
in non-hospital-based alternatives. The impact of stigma and a lack of wider medical training on psychiatric wards may account for the perceived delays in obtaining treatment for patients with physical health complaints admitted to psychiatric wards, whereas non-clinical staff or staff in non-clinical settings might have been more proactive in seeking help from primary care resources.

One fundamental difference that could be readily observed between the different types of services was their environments. Much has been made of the environment in which patients are treated..$^{20}$ Many changes have had a positive impact on patient care, such as the removal of ligature points. However, patients in this study demonstrated that there are diverse opinions and preferences for different types of services. Modern, 'state of the art' hospitals were valued equally with older buildings and those situated in converted buildings within the community. Environments that fostered positive interaction while maintaining people's need for privacy and space were optimal.

\section{Lessons in the provision of care}

How care was provided emerged as being as important as what was done in providing care to people admitted for acute mental health problems, especially given the absence of clear-cut differences in clinical outcomes for alternative compared with traditional services. ${ }^{21}$ How an aspect of care was provided frequently affected whether it was experienced positively or negatively by the patient. Relationships are an inherent influence in life and are the most important factor in defining the in-patient experience. $^{6}$ The majority of themes in this paper were expressed within the context of a relationship. The culture of relationships both on and off the ward has an important role, yet the relationships between staff and patients in in-patient services remains key. ${ }^{22}$ Gross abuses of trust in terms of sexual and physical abuse were rarely reported, but in everyday interactions both positive and negative relationships were described in each type of service. There was no defining difference in the quality of the relationships experienced between services. Positive relationships were most often defined in terms of effective communication, expressed in terms of caring, compassionate and helpful staff. Communication and communication style had further consequences in the provision of care. Actual coercion such as forcible medication may serve a useful purpose in some circumstances by benefiting the safety of both staff and the majority of patients on a ward. In contrast, soft coercion such as threats and perceived force were more widely used and described as equally pervasive, yet were often counterproductive. Soft coercion was more common in hospital-based services, and may be a further consequence of working with an acutely ill patient group. However, it was also present in alternative services, and may indicate a lack of effective communication skills in working with patients.

In maintaining safety and supporting recovery through a focus on the best interests of the patient, staff take on not only a caring role but often a custodial role. As a consequence staff can often be perceived as paternalistic. Patients identified the positive and negative impacts of paternalistic communication styles, which used effectively in a supportive role were experienced positively yet used to restrict and punish patients were uniformly experienced negatively. In the move towards deinstitutionalisation, service users value paternalistic input moving them closer towards recovery yet those that enforce rules characteristic of old institutions are counterproductive.

With widespread reports of poor experiences by Black and minority ethnic service users in psychiatric hospitals, ${ }^{23}$ alternative services specifically targeting these populations present a new and innovative way of providing acute mental healthcare. Such services are more oriented to cultural needs and the problems experienced by these communities. Our results suggest that such considerations are welcome but that the problems of working with marginalised communities may lie not singularly in providing culturally specific services but in working with staff to enhance cultural understanding and further consideration of patient-centred care provision.

\section{Methodological issues}

The study had some methodological limitations. Most importantly, patients were interviewed while residing at an alternative service. Their preferences and experiences may have been influenced by their current perceived need or lack of need for care from the service to which they had been admitted. Furthermore, retrospective recollection of their experiences of traditional hospital services may also be subject to other influences. However, patients' experiences in the alternative service in which the Tidal Model was previously implemented were similar in thematic content to those of traditional hospital services, suggesting that the influence of time on recollection of experiences was not large. Although the sample represented is large, it is unlikely to be representative. Only patients who were prepared to participate in research and in an interview with a researcher were included. Moreover, the study was conducted with a small number of alternative services, and patients in alternative services elsewhere may have had different experiences, whereas the views of patients who might have had poor experiences of alternative services and chosen not to return are not included in this study. The study placed users' views and testimonies at the centre of the methodological approach. In the vast majority of cases the researchers believed the participants' accounts to be accurate and pertinent. Mental healthcare workers might have expressed different views and stated good reasons for the similarities and differences in care that were identified by patients, but their views were not assessed in this study.

\section{Implications of the study}

In targeting a population who require episodes of care over their life but present a low risk to others during these periods, providing care in an alternative setting can be recommended. Alternative services were associated with optimising freedom, safety and patient interactions, and reducing levels of coercion, leading to better patient experience and satisfaction. Services with increased links into the community offering a level of continuity and improved patient experiences may instigate early help-seeking and early discharge, hence lessening the burden on current hospital acute care provision. Furthermore, in enhancing links with the community and social inclusion by giving patients greater levels of freedom and reducing unhelpful paternalism, potential institutionalisation is prevented. However, the implications of this research go beyond the values of alternative services. Hospital-based provision remains the mainstay of acute in-patient provision, ${ }^{5}$ and arguably is an essential component of an effective care system for a small number of people with acute mental illness. The selective admission of patients to alternative services may have an impact on hospital services by concentrating patients with high levels of disturbance, distress and risk. As has been identified by participants in this study, such environments present problems with regard to safety and the ability to engage and work therapeutically. There remains the question of how to create environments that contain and yet promote recovery in the least restrictive manner. 
Our research highlights the importance of considering not just the technical details of what care is provided, but also the interpersonal skills and personal qualities of the workforce - the 'how it is done' aspect. Key research challenges include enhancing therapeutic relationships, understanding effective communication, and supporting staff in providing care while maintaining a safe and therapeutic environment. Measures of patient satisfaction provide important evidence of the wide-scale impact of care and innovations, ${ }^{24}$ but can say little about the value placed on individual factors by patients. This points to the need for mixed-method studies when evaluating services, with the aim of establishing effective, acceptable and patient-centred care in health settings.

Helen Gilburt, PhD, Mike Slade, PhD, Health Service and Population Research Department, Institute of Psychiatry, King's College London; Diana Rose, PhD, Health Service and Population Research Department, Institute of Psychiatry, King's College London; Brynmor Lloyd-Evans, PhD, Sonia Johnson, DM, David P. J. Osborn, PhD, Department of Mental Health Sciences, University College London, UK

Correspondence: Dr Helen Gilburt, National Addictions Centre, PO Box 48, Institute of Psychiatry, King's College London, London SE5 8BB. Email: helen.gilburt@iop.kcl.ac.uk

\section{Funding}

This project was funded by the National Institute for Health Research Service Delivery and Organisation programme (project number 08/1304/075).

\section{Acknowledgements}

This study was undertaken in the context of the NIHR Specialist Mental Health Biomedica Research Centre at the Institute of Psychiatry, King's College London and the South London and Maudsley NHS Foundation Trust. The study was supported by the Mental Health Research Network (MHRN) and associated with the MHRN acute care group (convenor S.J.). The views expressed in this paper are those of the authors and not necessarily those of the NHS, the NIHR or the Department of Health.

\section{References}

1 Bowers L, Chaplin R, Quirk A, Lelliot P. A conceptual model of the aims and functions of acute inpatient psychiatry. J Ment Health 2009; 1: 1-10.

2 Quirk A, Lelliott $P$. What do we know about life on acute psychiatric wards in the UK? A review of research evidence. Soc Sci Med 2001; 53: 1565-74.

3 Relton P, Thomas P. Acute wards: problems and solutions. Alternatives to acute wards: users' perspectives. Psychiatr Bull 2002; 26: 346-7.

4 Sayce L, Christie Y, Cobb A, Slade M. Users' perspectives on emergency needs. In Emergency Mental Health Services in the Community (eds M Phelan, G Strathdee, G Thornicroft): 39-60. Cambridge University Press, 1994.

5 Barker P, Buchanan-Barker P. The Tidal Model: A Guide for Mental Health Professionals. Brunner-Routledge, 2005.
6 Johnson S, Gilburt H, Lloyd-Evans B, Osborn DPJ, Boardman J, Leese M, et al. In-patient and residential alternatives to standard acute psychiatric wards in England. Br J Psychiatry 2009; 194: 456-63.

7 Gilburt $H$, Rose $D$, Slade $M$. The importance of relationships in mental health care: a qualitative study of service users' experiences of psychiatric hospital admission in the UK. BMC Health Serv Res 2008; 8: 92.

8 Boyatzis RE. Transforming Qualitative Information: Thematic Analysis and Code Development. Sage, 1998.

9 Flick U. An Introduction to Qualitative Research. Sage, 2002.

10 Johnson S, Lloyd-Evans B, Morant N, Gilburt H, Shepherd G, Slade M, et al. Alternatives to acute in-patient care in England: roles and populations served. Br J Psychiatry 2010 (suppl 53): s6-13.

11 Johnson S, Gilburt H, Lloyd-Evans B, Slade M. Acute in-patient psychiatry: residential alternatives to hospital admission. Psychiatr Bull 2007; 31: 262-4.

12 Howard LM, Rigon E, Cole L, Lawlor C, Johnson S. Admissions to women's crisis houses or to psychiatric wards: women's pathways to admission. Psychiatr Serv 2008; 59: 1443-9.

13 Bola JR, Mosher LR. Predicting drug-free treatment response in acute psychosis from the Soteria project. Schizophr Bull 2002; 28: 559-75.

14 Johnson S, Bingham C, Billings J, Pilling S, Morant N, Bebbington $\mathrm{P}$, et al. Women's experiences of admission to a crisis house and to acute hospital wards: a qualitative study. J Ment Health 2004; 13: 247-62.

15 Keown P, Mercer G, Scott J. Retrospective analysis of hospital episode statistics, involuntary admissions under the Mental Health Act 1983, and number of psychiatric beds in England 1996-2006. BMJ 2008; 337: 1837.

16 Bowers L, Allan T, Simpson A, Jones J, Van der Merwe M, Jeffery D. Identifying key factors associated with aggression on acute inpatient psychiatric wards. Issues Ment Health Nurs 2009; 20: 260-71.

17 Kumar S, Guite H, Thornicroft G. Service users' experience of violence within a mental health system: a study using grounded theory approach. J Ment Health 2001; 10: 597-611.

18 Commission of Healthcare Audit and Inspection. The Pathway to Recovery: A Review of NHS Acute Inpatient Mental Health Services. Commission for Healthcare Audit and Inspection, 2008.

19 Lloyd-Evans B, Johnson S, Morant N, Gilburt H, Osborn DPJ, Jagielska D, et al. Alternatives to standard acute in-patient care in England: differences in content of care and staff-patient contact. Br J Psychiatry 2010 (suppl 53): s46-51.

20 Karlin BE, Zeiss RA. Environmental and therapeutic issues in psychiatric hospital design: towards best practices. Psychiatr Serv 2006; 57: 1376-8.

21 Slade M, Byford S, Barrett B, Lloyd-Evans B, Gilburt H, Osborn DPJ, et al. Alternatives to standard acute in-patient care in England: short-term clinical outcomes and cost-effectiveness. Br J Psychiatry 2010 (suppl 53): s14-9.

22 Walsh J, Boyle J. Improving acute psychiatric hospital services according to inpatient experiences. A user-led piece of research as a means to empowerment. Issues Ment Health Nurs 2009; 30: 31-8.

23 Francis E, David J, Johnson N, Sashidharan SP. Black people and psychiatry in the UK: an alternative to institutional care. Psychiatr Bull 1989; 13: 482-5.

24 Osborn DPJ, Lloyd-Evans B, Johnson S, Gilburt H, Byford S, Leese M, et al. Residential alternatives to acute in-patient care in England: satisfaction, ward atmosphere and service user experiences. Br J Psychiatry 2010 (suppl 53): s41-5. 